A. Conca

Nagoya Math. J.

Vol. 136 (1994), 35-56

\title{
SYMMETRIC LADDERS
}

\author{
ALDO CONCA
}

In this paper we define and study ladder determinantal rings of a symmetric matrix of indeterminates. We show that they are Cohen-Macaulay domains. We give a combinatorial characterization of their $h$-vectors and we compute the $a$-invariant of the classical determinantal rings of a symmetric matrix of indeterminates.

\section{Introduction}

Let us recall the definition of ladder determinantal rings of a generic matrix of indeterminates. Let $X$ be a generic matrix of indeterminates, $K$ be a field and denote by $K[X]$ the polynomial ring in the set of indeterminates $X_{i j}$. A subset $Y$ of $X$ is called a ladder if whenever $X_{i j}, X_{h k} \in Y$ and $i \leq h, j \leq k$, then $X_{i k}, X_{h j} \in Y$. Given a ladder $Y$, one defines $I_{t}(Y)$ to be the ideal generated by all the $t$-minors of $X$ which involve only indeterminates of $Y$. The ideal $I_{t}(Y)$ is called a ladder determinantal ideal and the quotient $R_{t}(Y)=K[Y] / I_{t}(Y)$ is called a ladder determinantal ring. This class of ideals is investigated in [1], [2], [9], [15], [17]. It turns out that the main tool in the investigation of the ladder determinantal rings is the knowledge of Gröbner bases of the classical determinantal ideals. In [8] we determined Gröbner bases of ideals generated by minors of a symmetric matrix of indeterminates. This allows us to study the ladder determinantal rings of a symmetric matrix.

Now let $X$ be an $n \times n$ symmetric matrix of indeterminates, $K$ be a field. Let us denote by $A$ the set $\left\{(i, j) \in \mathbf{N}^{2}: 1 \leq i, j \leq n\right\}$. A subset $L$ of $A$ is called a symmetric ladder if satisfies the following condition: if $(i, j) \in L$ then $(j, i) \in$ $L$, and whenever $(i, j),(h, k) \in L$ and $i \leq h, j \leq k$, then $(i, k),(h, j) \in L$.

The set $Y=\left\{X_{i j}: i \leq j,(i, j) \in L\right\}$ is called the support of $L$. We say that a minor is in $L$ if it involves only indeterminates of $Y$. Given a sequence of integers $\alpha=1 \leq \alpha_{1}<\cdots<\alpha_{t} \leq n$, we define $I_{\alpha}(L)$ to be the ideals generated

Received April 6, 1993. 
by all the $i$-minors of the first $\alpha_{i}-1$ rows of $X$ which are in $L, i=1, \ldots, t$, and by all the $t+1$ minors of $L$. Denote by $R_{\alpha}(L)$ the ring $K[Y] / I_{\alpha}(L)$. In particular if $\alpha=1, \ldots, t-1$, then $I_{\alpha}(L)$ is the ideal generated by the $t$-minors in $L$.

Following the approach of Narasimhan [17], we use Gröbner bases to show that $I_{\alpha}(L)=I_{\alpha}(X) \cap K[Y]$. Since $I_{\alpha}(X)$ is known to be a prime ideal, see [16], it follows that $I_{\alpha}(L)$ is prime too. Furthermore we determine a Gröbner basis of the ideal $I_{\alpha}(L)$. It turns out that the ideal in $\left(I_{\alpha}(L)\right)$ of the leading forms of $I_{\alpha}(L)$ is generated by square free monomials. Therefore the ring $R_{\alpha}(L)^{*}=K[Y] /$ in $\left(I_{\alpha}(L)\right)$ is the Stanley-Reisner ring associated with a simplicial complex $\Delta_{\alpha}(L)$. By a result of Stanley, the Hilbert function of $R_{\alpha}(L)^{*}$ is determinated by the $f$-vector of $\Delta_{\alpha}(L)$. We describe the facets of $\Delta_{\alpha}(L)$ in terms of families of non-intersecting chains in a poset, and we get a combinatorial characterization of the dimension and multiplicity of $R_{\alpha}(L)$. As in the case of ladders of a generic matrix, it is possible to show that $\Delta_{\alpha}(L)$ is shellable. Actually, we deduce this result from the analogous of [15]. The shellability is a combinatorial property of simplicial complexes which implies the Cohen-Macaulayness of the associated Stanley-Reisner rings. But it is well known that if $R_{\alpha}(L)^{*}$ is Cohen-Macaulay, then $R_{\alpha}(L)$ is.

In the second section we apply these results to give a combinatorial characterization of the $h$-vector of the rings $R_{\alpha}(X)$ in terms of number of families of non-intersecting paths in a poset with a fixed number of certain corners. Then we compute the $a$-invariant of the ring $R_{t}(X)$ defined by the ideal of minors of fixed size in the matrix $X$ in the homogeneous and weighted case. The same result was obtained by Barile [3] independently and using different methods. As last application we study the determinantal ring $R_{t}(Z)$ associated with an $m \times n$ matrix of indeterminates $Z$ in which an $s \times s$ submatrix is symmetric. It turns out that $R_{t}(Z)$ is a symmetric ladder determinantal ring. In particular $R_{t}(Z)$ is a Cohen-Macaulay domain, and we compute its dimension and multiplicity. If $s<m$ $\leq n$, we prove that $R_{t}(Z)$ is normal and that is Gorenstein if and only if $t \geq s$ and $m=n$. In [10] we deal with the case $s=m<n$, and we show that $R_{t}(Z)$ is normal, and is Gorenstein if and only if $2 m=n+t$. The results of this paper are part of the author's $\mathrm{Ph}$. D. thesis.

\section{Ladders of a symmetric matrix}

Let $X$ be an $n \times n$ symmetric matrix of indeterminates, $K$ be a field, and denote by $K[X]$ the polynomial ring in the set of indeterminates $X_{i j}, 1 \leq i \leq j \leq n$. Let $\tau$ be the term order induced by the variable order $X_{11}>\cdots>X_{1 n}>X_{22}>$ 


\section{$\cdots>X_{2 n}>\cdots>X_{n-1 n}>X_{n n}$}

Let us recall the combinatorial structure of $K[X]$ with respect to the product of minors of $X$. Denote by $H$ the set of the non-empty subsets of $\{1, \ldots, n\}$. Given an element $a$ of $H$ we will always write its elements in ascending order $1 \leq a_{1}<\cdots<a_{s} \leq n$. On $H$ we define the following partial order:

$$
a=\left\{a_{1}, \ldots, a_{s}\right\} \leq b=\left\{b_{1}, \ldots, b_{r}\right\} \Leftrightarrow r \leq s \text { and } a_{i} \leq b_{i} \text { for } i=1, \ldots, r .
$$

As usual, we denote by $\left[a_{1}, \ldots, a_{s} \mid b_{1}, \ldots, b_{s}\right]$ the $s$-minor $\operatorname{det}\left(X_{a_{i} b_{j}}\right)$ of $X$, and assume that $1 \leq a_{1}<\cdots<a_{s} \leq n$ and $1 \leq b_{1}<\cdots<b_{s} \leq n$. The minor $\left[a_{1}, \ldots, a_{s} \mid b_{1}, \ldots, b_{s}\right]$ is called a doset minor if $a \leq b$ in $H$. We denote by $D$ the set of all the doset minors of $X$. Let $M_{1}=\left[a_{11}, \ldots, a_{1 s_{1}} \mid b_{11}, \ldots, b_{1 s_{1}}\right], \ldots, M_{p}=$ $\left[a_{p 1}, \ldots, a_{p s_{p}} \mid b_{p 1}, \ldots, b_{p s_{p}}\right]$ be doset minors; the product $M_{1} \cdots M_{p}$ is called a standard monomial if $\left\{b_{j 1}, \ldots, b_{j s,}\right\} \leq\left\{a_{j+11}, \ldots, a_{j+1 s_{j+1}}\right\}$ for $j=1, \ldots, p-1$. The ring $K[X]$ is a doset algebra on $D$, that is, the standard monomials form a $K$-basis of $K[X]$ and one has a certain control on the miltiplicative table of the products of the standard monomials, see [12]. If one considers suitable ideals of minors, the same combinatorial structure is inherited by the quotient rings. Given $\alpha=\left\{\alpha_{1}, \ldots, \alpha_{t}\right\} \in H$ one defines $I_{\alpha}(X)$ to be the ideal generated by all the minors $\left[a_{1}, \ldots, a_{s} \mid b_{1}, \ldots, b_{s}\right]$ with $\left\{\alpha_{1}, \ldots, \alpha_{s}\right\} \ngtr \alpha$ in $H$. If $\alpha=\{1, \ldots, t-1\}$, then the ideal $I_{\alpha}(X)$ is the ideal $I_{t}(X)$ generated by all the $t$-minors of $X$. The class of ideals $I_{\alpha}(X)$ is essentially the same the class of ideals defined and studied by Kutz [16].

In order to define ladders and ladder determinantal ideals of the symmetric matrix $X$ we introduce some notations. Let $A=\left\{(i, j) \in \mathbf{N}^{2}: 1 \leq i \leq n\right.$ and $1 \leq j \leq n\}$ and $B=\{(i, j) \in A: i \leq j\}$. We consider $A$ a distributive lattice with the following partial order: $(i, j) \leq(k, h) \Leftrightarrow i \geq k$ and $j \leq h$.

In the generic case there is a one-to-one correspondence between minors and monomials which are product of elements of main diagonals of minors. When we deal with minors of a symmetric matrix we lose this correspondence. The monomial $X_{a_{1} b_{1}} \ldots X_{a_{s} b_{s}}$, with $a_{i}<a_{i+1}$ and $b_{i}<b_{i+1}$, is the product of the elements on the main diagonal of all the minors $M=\left[c_{1}, \ldots, c_{s} \mid d_{1}, \ldots, d_{s}\right]$ such that $\left\{c_{i}\right.$, $\left.d_{i}\right\}=\left\{a_{i}, b_{i}\right\}$ and $c_{i}<c_{i+1}, d_{i}<d_{i+1}$. But if we require that the minor is a doset minor then it is unique.

Therefore the natural choice for the definition of a ladder of the symmetric matrix $X$ is the following:

Definition 1.1. A subset $L$ of $A$ is a symmetric ladder if:

(a) $L$ is a sublattice of $A$; 
(b) $L$ is symmetric, that is $(i, j) \in L$ if and only if $(j, i) \in L$.

We represent ladders as subsets of points of $\mathbf{N}^{2}$. An example of symmetric ladder is the following:

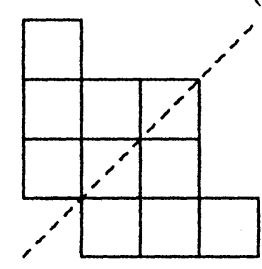

$(1,1)$

Fig. 1

Let $L$ be a symmetric ladder, we put $L^{+}=L \cap B$ and $Y=\left\{X_{i j}:(i, j) \in\right.$ $L, i \leq j\}$. The set $Y$ is called the support of $L$. We say that a minor $M=\left[a_{1}, \ldots\right.$, $\left.a_{s} \mid b_{1}, \ldots, b_{s}\right]$ is in $L$ if the following equivalent conditions are satisfied:

(1) For all $1 \leq i, j \leq s$, then $\left(a_{i}, b_{j}\right) \in L$.

(2) For all $1 \leq i \leq s$, then $\left(a_{i}, b_{i}\right) \in L$.

(3) The entries of $M$ belong to $Y$.

(4) The entries of the main diagonal of $M$ belong to $Y$.

Let $\alpha=\left\{\alpha_{1}, \ldots, \alpha_{t}\right\} \in H$. For systematic reasons it is convenient to set $\alpha_{t+1}=n+1$. Following [15], we define the ideal cogenerated by $\alpha$ in $L$.

Definition 1.2. Let $L$ be a symmetric ladder and $Y$ its support. We denote by $I_{\alpha}(L)$ the ideal generated by all the minors $M=\left[a_{1}, \ldots, a_{s} \mid b_{1}, \ldots, b_{s}\right]$ of $L$ such that $\left\{a_{1}, \ldots, a_{s}\right\} \geq \alpha$ and set $R_{\alpha}(L)=K[Y] / I_{\alpha}(L)$.

In particular, if $\alpha=\{1, \ldots, t-1\}$, then $I_{\alpha}(L)$ is the ideal generated by all the $t$-minors of $L$.

Let $J_{\alpha}(L)$ be the set of all the doset minors $\left[a_{1}, \ldots, a_{r} \mid b_{1}, \ldots, b_{r}\right]$ of $L$ such that $1 \leq r \leq t+1, a_{i} \geq \alpha_{i}$ for $i=1, \ldots, r-1$ and $a_{r}<\alpha_{r}$. The main result of [8] is the determination of a Gröbner basis of the ideal $I_{\alpha}(X)$ with respect to $\tau$ : the set $J_{\alpha}(X)$ is a minimal system of generators and a Gröbner basis with respect to $\tau$ of the ideal $I_{\alpha}(X)$, see $[8,2.7,2.8]$. From this we deduce the following:

THEOREM 1.3. (a) The ideal $I_{\alpha}(L)$ is prime.

(b) The set $J_{\alpha}(L)$ is a Gröbner basis of $I_{\alpha}(L)$ with respect to $\tau$.

(c) The set $J_{\alpha}(L)$ is a minimal system of generators of $I_{\alpha}(L)$. 
Proof. (a) Since $I_{\alpha}(X)$ is a prime ideal, see [16, Th. 1], it is sufficient to show that $I_{\alpha}(L)=I_{\alpha}(X) \cap K[Y]$. We have $I_{\alpha}(L) \subset I_{\alpha}(X) \cap K[Y]$ since, by definition, $I_{\alpha}(L) \subset I_{\alpha}(X)$. Let $f \in I_{\alpha}(X) \cap K[Y]$ be an homogeneous polynomial and denote by $\operatorname{in}(f)$ its initial term with respect to $\tau$. The set $J_{\alpha}(X)$ is a Gröbner basis of $I_{\alpha}(X)$. Therefore in $(f)$ is divisible by the initial term of a doset minor $M$ of $J_{\alpha}(X)$, that is, in $(f)=\operatorname{in}(M) h$. Of course in $(f) \in K[Y]$, and therefore the minor $M$ is in $L$. Note that $M \in J_{\alpha}(L)$. Set $g=f-h M$; then we have $g \in$ $I_{\alpha}(X) \cap K[Y]$ and $g=0$ or in $(g)<$ in $(f)$ in the term ordering. Therefore, by induction, we may suppose $g \in I_{\alpha}(L)$ and $f=g+h M \in I_{\alpha}(L)$.

(b) Let $f \in I_{\alpha}(L)$, since in $(f) \in \operatorname{in}\left(I_{\alpha}(X)\right) \cap K[Y]$ we may argue as in the proof of part (a) and show that in $(f)$ is divisible by the initial term of a minor of $J_{\alpha}(L)$.

(c) Since $J_{\alpha}(L)$ is a Gröbner basis of $I_{\alpha}(L)$, it is also a system of generators. But $J_{\alpha}(X)$ is a minimal system of generators of $I_{\alpha}(X)$ and $J_{\alpha}(L) \subset J_{\alpha}(X)$. Therefore $J_{\alpha}(L)$ is a minimal system of generators of $I_{\alpha}(L)$.

Now we see how we may interpret the ideal $I_{\alpha}(L)$ as an ideal of minors associated with more general subsets of $A$.

Definition 1.4. A subset $V$ of $A$ is a semi-symmetric ladder if:

(a) $V$ is a sublattice of $A$.

(b) If $(i, j) \in V$ and $i \geq j$, then $(j, i) \in V$.

Given a semi-symmetric ladder $V$, we say that a minor $\left[a_{1}, \ldots, a_{s} \mid b_{1}, \ldots, b_{s}\right]$ is in $V$ if $\left(a_{i}, b_{j}\right) \in V$ for all $1 \leq i, j \leq s$. We define $I_{\alpha}(V)$ to be the ideal generated of all minors in $V$ whose sequence of row indices is not greater than or equal to $\alpha$.

Remark 1.5. Let $V$ be a semi-symmetric ladder and set $L(V)=\{(i, j) \in A$ : $(i, j) \in V$ or $(j, i) \in V\}$. It is easy to see that $L(V)$ is a symmetric ladder and that $L(V)^{+} \subset V$. If we consider a doset minor $M$ in $L(V)$, then its main diagonal is in $L(V)^{+}$, and therefore $M$ is in $V$. Hence $I_{\alpha}(V)=I_{\alpha}(L(V))$. In other words, to study ideals of minors of symmetric ladders is the same as to study ideals of minors of semi-symmetric ladders.

In the picture $V$ is a semi-symmetric ladder and $L(V)$ is its associated symmetric ladder. 

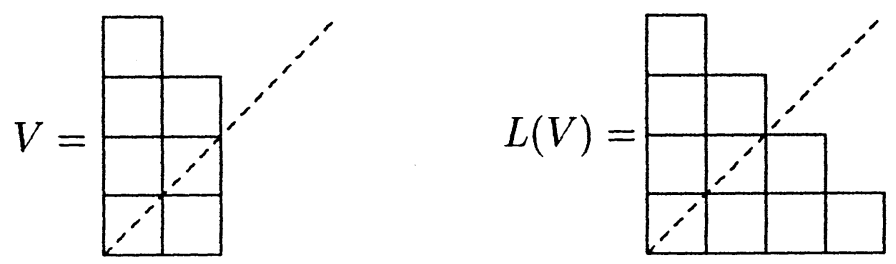

Fig. 2

The ideal in $\left(I_{\alpha}(L)\right)$ of the leading forms of $I_{\alpha}(L)$ is generated by the leading terms of the minors in $J_{\alpha}(L)$, and hence it is a square-free monomial ideal. Therefore $R_{\alpha}(L)^{*}=K[Y] /$ in $\left(I_{\alpha}(L)\right)$ is the Stanley-Reisner ring associated with a simplicial complex. For the theory of the Stanley-Reisner ring associated with a simplicial complex we refer the reader to [18].

In order to describe this simplicial complex and its facets we introduce some notation and terminology. Given a simplicial complex $\Delta$, its elements are called faces and facets its maximal elements under inclusion. A face of dimension $i$ is a face with $i+1$ elements, the dimension of $\Delta$ is the maximum of the dimensions of its faces and $f_{i}$ is the number of the faces of dimension $i$. The sequence $f_{0}, \ldots, f_{d}$, $d=\operatorname{dim}(\Delta)$, is called the $f$-vector of $\Delta$. The Hilbert function of the StanleyReisner ring $k[\Delta]$ is determined by its $f$-vector [18]. In particular, $\operatorname{dim} k[\Delta]=d+1$ and $e(k[\Delta])=f_{d}$.

Let $P$ be a finite poset and $x \in P$. We define the rank of $x$ in $P$ to be the maximum of the integers $i$ such that there exists a chain $x_{1}<\cdots<x_{i}=x$ and the rank of $P$ to be the maximum of the ranks of its elements. A set of incomparable elements of $P$ is called an antichain. An antichain of $B$ is a set $\left\{\left(v_{1}, u_{1}\right), \ldots\right.$, $\left.\left(v_{p}, u_{p}\right)\right\}$ with $v_{i} \leq u_{i}$ for $i=1, \ldots, p$ such that $v_{1}<\cdots<v_{p}$ and $u_{1}<\cdots$ $<u_{p}$ and therefore it corresponds to the main diagonal of a doset minor.

For $k=1, \ldots, t+1$, let $S_{k}=\left\{(i, j) \in A: i<\alpha_{k}\right.$ or $\left.j<\alpha_{k}\right\}, G_{k}=B \cap$ $S_{k}, S_{k}^{\prime}=A \backslash S_{k}$ and $G_{k}^{\prime}=B \backslash G_{k}$.

We define $\Delta_{\alpha}^{\prime}(L)$ to be the simplicial complex of all the subsets of $L$ which, for $k=1, \ldots, t+1$, do not contain $k$-antichains (antichains with $k$ elements) of $S_{k} \cap L$, and let $\Delta_{\alpha}(L)$ be the restriction of $\Delta_{\alpha}^{\prime}(L)$ to $L^{+}$. By construction $\Delta_{\alpha}(L)$ is the simplicial complex of all the subsets of $L^{+}$which, for $k=1, \ldots, t+1$, do not contain $k$-antichains of $G_{k} \cap L^{+}$. Furthermore the simplicial complex $\Delta_{\alpha}^{\prime}(L)$ coincides with the simplicial complex $\Delta_{M}(L)$ defined in [15], where $M=\left[\alpha_{1}, \ldots\right.$, $\left.\alpha_{t} \mid \alpha_{1}, \ldots, \alpha_{t}\right]$. 
We know that in $\left(I_{\alpha}(L)\right)$ is generated by the $k$-antichains of $G_{k} \cap L^{+}$for $k=1, \ldots, t+1$. Therefore the Stanley-Reisner ring $K\left[\Delta_{\alpha}(L)\right]$ associated with $\Delta_{\alpha}(L)$ is $R_{\alpha}(L)^{*}$.

It is well known that $R_{\alpha}(L)$ and $R_{\alpha}(L)^{*}$ have the same Hilbert series, therefore their dimensions and multiplicities coincide. Thus the Hilbert function, the multiplicity, and the dimension of $R_{\alpha}(L)$ may be characterized in terms of $f$-vector of $\Delta_{\alpha}(L)$.

Let $p=(a, b)$ be an element of $L$, we define $R_{p}=\{(i, j) \in L: a<i, b$ $<j$, and for $Z \subset L$ we set $R_{Z}=\cup_{p \in Z} R_{p}$. It is easy to see that $R_{Z}$ is a sublattice of $L$ which is symmetric if $Z$ is. We set $L_{1}=L \cap S_{1}$ and recursively for $i=2, \ldots, t$, we set $L_{i}=S_{i} \cap R_{L_{i-1}}$. Finally we put $L_{i}^{+}=L_{i} \cap B$. Since $S_{1}$ and $L$ are symmetric sublattices of $A, L_{1}$ is also a symmetric sublattice of $A$. By recursion it follows that $L_{\imath}$ is the intersection of symmetric sublattices, and therefore it is also a symmetric sublattice.

By [15, Th. 4.6] a subset $\bar{Z}$ of $L$ is a facet of $\Delta_{\alpha}^{\prime}(L)$ if and only if $\bar{Z}$ is the union of disjoint maximal chains of $L_{i}, i=1, \ldots, t$.

Lemma 1.6. Let $\bar{Z}$ be a facet of $\Delta_{\alpha}^{\prime}(L)$, then $\left|\bar{Z} \cap L^{+}\right|=\sum_{i=1}^{t} r k\left(L_{i}^{+}\right)$where $r k\left(L_{i}^{+}\right)$is the rank of the poset $L_{i}^{+}$.

Proof. Let $p \in L_{i}$, we claim: $p \in L_{i}^{+} \Leftrightarrow \operatorname{rk}(p)>\left[\operatorname{rk}\left(L_{i}\right) / 2\right]$, where $\operatorname{rk}(p)$ is the rank of $p$ in the lattice $L_{i}$, and $[x]=\max \{n \in \mathbf{Z}: n \leq x\}$ denote the integer part of a real number $x$.

$\Rightarrow$ : Let $p_{1}<\cdots<p_{s}$ be a maximal chain of $L_{i}^{+}$which contains $p$, say $p=$ $p_{k}$. If we consider the sequence $q_{1}, \ldots, q_{s}$ of the symmetric points $\left(q_{j}\right.$ is obtained from $p_{j}$ by exchanging the coordinates), then $q_{s}<\cdots<q_{2}<q_{1} \leq p_{1}<p_{2}<\cdots$ $<p_{s}$ is a maximal chain of $L_{i}$. Since $L_{i}$ is a distributive lattice and all the maximal chains of a distributive lattice have the same number of elements, we have $\operatorname{rk}\left(L_{i}\right)=2 s$ if $p_{1} \neq q_{1}$, and $\operatorname{rk}\left(L_{i}\right)=2 s-1$ if $p_{1}=q_{1}$. In any case $\operatorname{rk}(p) \geq$ $\operatorname{rk}\left(p_{1}\right)>\left[\operatorname{rk}\left(L_{i}\right) / 2\right]$.

$\Leftarrow$ : Suppose $p \notin L_{i}^{+}$and let $q_{1}<\cdots<q_{k}=p$ be a chain with $k=\operatorname{rk}(p)$ elements. If we consider the sequence of the symmetric points $p_{1}, \ldots, p_{k}$ then $q_{1}<\cdots<q_{k}<p_{k}<\ldots<p_{1}$ is a chain of $L_{i}$ with $2 \operatorname{rk}(p)$ elements Therefore $\mathrm{rk}\left(L_{i}\right) \geq 2 \mathrm{rk}(p)>2\left[\mathrm{rk}\left(L_{i}\right) / 2\right]$, a contradiction.

From the previous claim it follows that every maximal chain of $L_{i}$ contains exactly $\operatorname{rk}\left(L_{i}\right)-\left[\operatorname{rk}\left(L_{\imath}\right) / 2\right]$ elements of $L_{\imath}^{+}$and $\operatorname{rk}\left(L_{i}^{+}\right)=\operatorname{rk}\left(L_{\imath}\right)-\left[\operatorname{rk}\left(L_{i}\right) / 2\right]$. Hence the assertion of the lemma follows from the description of the facets of $\Delta_{\alpha}^{\prime}(L)$ and the claim. 
As immediate consequence we get:

Proposition 1.7. Let $Z$ be a face of $\Delta_{\alpha}(L)$. Then $Z$ is a facet of $\Delta_{\alpha}(L)$ if and only if there exists a facet $\bar{Z}$ of $\Delta_{\alpha}^{\prime}(L)$ such that $Z=\bar{Z} \cap L^{+}$.

Proof. $\Rightarrow$ : The simplicial complex $\Delta_{\alpha}(L)$ is the restriction of the simplicial complex $\Delta_{\alpha}^{\prime}(L)$ to $L^{+}$. Therefore there exists a facet $\bar{Z}$ of $\Delta_{\alpha}^{\prime}(L)$ such that $Z \subset \bar{Z}$ $\cap L^{+}$. Since $Z$ is a facet, $Z=\bar{Z} \cap L^{+}$.

$\Leftarrow$ : Of course $Z$ is contained in a facet $Z_{1}$ of $\Delta_{\alpha}(L)$. By 1.6 it follows that $|Z|=$ $\left|Z_{1}\right|$, and hence $Z=Z_{1}$.

We get the following characterization of the facets of $\Delta_{\alpha}(L)$ :

PROPOSITION 1.8. The set $Z$ is a facet of $\Delta_{\alpha}(L)$ if and only if $Z$ is the union of disjoint sets $Z_{1}, \ldots, Z_{t}$, where $Z_{i}$ is a maximal chain of $L_{i}^{+}$. Furthermore the decomposition of $Z$ as union of disjoint maximal chains of $L_{i}^{+}$is unique.

Proof. The set $Z$ is a facet of $\Delta_{\alpha}(L)$ if and only if there exists a facet $\bar{Z}$ of $\Delta_{\alpha}^{\prime}(L)$ such that $Z=\bar{Z} \cap L^{+}$. But $\bar{Z}$ is the union of disjoint sets $\bar{Z}_{1}, \ldots, \bar{Z}_{t}$, where $\bar{Z}_{i}$ is a maximal chain of $L_{i}$. If we set $Z_{i}=\bar{Z}_{i} \cap L_{i}^{+}$then $Z_{i}$ is a maximal chain of $L_{i}^{+}$and $Z$ is the union of $Z_{1}, \ldots, Z_{t}$. The uniqueness of the decomposition of $Z$ is a consequence of the construction of the decomposition of $\bar{Z}$ as union of disjoint maximal chains, see [15, pp. 20].

COROLLARY 1.9. The dimension of $R_{\alpha}(L)$ is $\sum_{i=1}^{t} \mathrm{rk}\left(L_{i}^{+}\right)$, and its multiplicity is the number of the families of disjoint sets $Z_{1}, \ldots, Z_{t}$, where $Z_{i}$ is a maximal chain of $L_{i}^{+}$.

Using this result we computed in [8] the dimension and the multiplicity of the ring $R_{\alpha}(X)$.

Recall that a simplicial complex $\Delta$ is said to be shellable if its facets have the same dimension and they can be given a linear order called a shelling in such a way that if $Z<Z_{1}$ are facets of $\Delta$, then there exists a facet $Z_{2}<Z_{1}$ of $\Delta$ and an element $x \in Z_{1}$ such that $Z \cap Z_{1} \subseteq Z_{2} \cap Z_{1}=Z_{1} \backslash\{x\}$.

By [15, Th. 4.9] the simplicial complex $\Delta_{\alpha}^{\prime}(L)$ is shellable. Now we shall see how shellability passes from a simplicial complex to a subcomplex when a condition as 1.6 is fulfilled. 
Lemma 1.10. Let $\Delta$ be a shellable simplicial complex over a vertices set $V$, and $W$ a subset of $V$. Suppose that for all the facets $\bar{Z}$ of $\Delta$ the number $|\bar{Z} \cap W|$ does not depend on $\bar{Z}$. Then the restriction of $\Delta$ to $W$ is a shellable simplicial complex.

Proof. We denote by $\Delta_{1}$ the restriction of $\Delta$ to $W, F(\Delta)$ the set of the facets of $\Delta, F\left(\Delta_{1}\right)$ the set of the facets of $\Delta_{1}, n=|\bar{Z} \cap W|$ for all $\bar{Z} \in F(\Delta)$.

From the hypotheses follows, as in 1.7 , that $\Delta_{1}$ is a pure simplicial complex of dimension $n-1$ and that a subset $Z$ of $W$ is in $F\left(\Delta_{1}\right)$ if and only if there exists $\bar{Z} \in F(\Delta)$ such that $\bar{Z} \cap W=Z$. If $Z \in F\left(\Delta_{1}\right)$, we define $Z^{\prime}=\min \{\bar{Z} \in F(\Delta)$ : $\bar{Z} \cap W=Z$, where the minimum is taken with respect to the total order of $F(\Delta)$. We define a total order on $F\left(\Delta_{1}\right)$ setting: $Z<Z_{1} \Leftrightarrow Z^{\prime}<Z_{1}^{\prime}$ in $F(\Delta)$, and show that this order gives the desired shelling.

Let $Z, Z_{1} \in F\left(\Delta_{1}\right)$ with $Z<Z_{1}$. By definition $Z^{\prime}<Z_{1}^{\prime}$ in $F(\Delta)$. Since the total order on $F(\Delta)$ is a shelling, there exists $H \in F(\Delta)$ and $x \in Z_{1}^{\prime}$ such that $H$ $<Z_{1}^{\prime},\{x\}=Z_{1}^{\prime} \backslash H$ and $Z_{1}^{\prime} \cap Z^{\prime} \subset Z_{1}^{\prime} \cap H$. We note that $x \in Z_{1}$ since otherwise $H \cap W=Z_{1}$ and $H<Z_{1}^{\prime}$, a contradiction with the definition of $Z_{1}^{\prime}$. Let $Z_{2}=H \cap$ $W ; Z_{2} \in F\left(\Delta_{1}\right)$ since $H \in F(\Delta),\{x\}=Z_{1} \backslash Z_{2}$ and $Z_{1} \cap Z \subset Z_{1} \cap Z_{2}$. By definition, $Z_{2}^{\prime} \leq H<Z_{1}^{\prime}$ and therefore $Z_{2}<Z_{1}$.

Let $H_{S}(t)$ be the Hilbert series of a homogeneous $K$-algabra $S$ (here the degrees of the generators are all 1). It is well-known that $H_{S}(t)=\sum_{i=0}^{s} h_{i} t^{i} /(1-$ $t)^{d}$, where $d$ is the dimension of $S, h_{i} \in \mathbf{Z}$, and $h_{s} \neq 0$. The vector $\left(h_{0}, \ldots, h_{s}\right)$ is called the $h$-vector of $S$. The McMullen-Walkup formula, see [5], is a combinatorial interpretation of the $h$-vector of the Stanley-Reisner ring associated with a shellable simplicial complex. Given a facet $Z_{1}$ of a shellable simplicial complex $\Delta$, we set

$$
C\left(Z_{1}\right)=\left\{x \in V: \text { there exists a facet } Z \text { of } \Delta \text { such that } Z<Z_{1} \text { and } Z_{1} \backslash Z=\{x\}\right\} .
$$

Let $\left(h_{0}, \ldots, h_{s}\right)$ be the $h$-vector of the Stanley-Reisner ring associated with $\Delta$. The McMullen-Walkup formula is:

$$
h_{i}=\mid\{Z \text { facet of } \Delta:|C(Z)|=i\} \mid \text {. }
$$

Under the assumption the previous lemma and with the notation introduced in the proof, we get:

Lemma 1.11. Let $Z_{1} \in F\left(\Delta_{1}\right)$, then $C\left(Z_{1}\right)=C\left(Z_{1}^{\prime}\right)$. 
Proof. Let $x \in C\left(Z_{1}\right)$, and $Z \in F\left(\Delta_{i}\right)$ such that $Z<Z_{1}$ and $Z_{1} \backslash Z=\{x\}$. Then $Z^{\prime}<Z_{1}^{\prime}$; there exist $H \in F(\Delta)$ and $y \in V$ such that $H<Z_{1}^{\prime}, Z^{\prime} \cap Z_{1}^{\prime} \subset H$ $\cap Z_{1}^{\prime}=Z_{1}^{\prime} \backslash\{y\}$. By definition of $Z_{1}^{\prime}$, the restriction of $H$ to $W$ is not $Z_{1}$. Therefore we get $y=x$, and $C\left(Z_{1}\right) \subset C\left(Z_{1}^{\prime}\right)$.

Conversely, let $y \in C\left(Z_{1}^{\prime}\right)$, and $H \in F(\Delta)$ such that $H<Z_{1}^{\prime}$ and $Z_{1}^{\prime} \backslash H=$ $\{y\}$. Again the restriction of $H$ to $W$ is not $Z_{1}$, and therefore $y \in W$. Let $Z=H \cap$ $W ; Z \in F\left(\Delta_{1}\right)$, and $Z<Z_{1}$ since $Z^{\prime} \leq H<Z_{1}^{\prime}$. Furthermore $Z_{1} \backslash Z=\{y\}$, and we are done.

Proposition 1.12. The simplicial complex $\Delta_{\alpha}(L)$ is shellable.

Proof. Straightforward by 1.6 and 1.10 .

The Stanley-Reisner ring associated with a shellable simplicial complex is Cohen-Macaulay, [4]. It is well-known that if $R_{\alpha}(L){ }^{*}$ is Cohen-Macaulay, then $R_{\alpha}(L)$ Cohen-Macaulay too, see for instance [14] or [6]. Therefore from the shellability of $\Delta_{\alpha}(L)$ we deduce the Cohen-Macaylayness of $R_{\alpha}(L)$. By $1.3, R_{\alpha}(L)$ is a domain, and we get the main theorem of this section:

Theorem 1.13. The ring $R_{\alpha}(L)$ is a Cohen-Macaulay domain.

In particular the previous theorem gives an alternative proof of the Cohen-Macaulayness of the ring $R_{\alpha}(X)$, see [16].

\section{Some applications}

We present some applications of the results of the first section. First, following the approach of [5] and [11], we give a combinatorial interpretation of the $h$-vector of the determinantal rings $R_{\alpha}(X)$ in terms of families of non-intersecting paths. Secondly, we compute the $a$-invariant of the determinantal rings $R_{t}(X)$ in the homogeneous and weighted case. The same formula was obtained, independently and using different methods, by Barile, see [3]. Finally we study, as an interesting class of symmetric ladder determinantal rings, the determinantal ring associated with a matrix of indeterminates in which a submatrix is symmetric.

\subsection{Characterization of the $h$-vector}

We keep the notation of the first section. The $h$-vector of $R_{\alpha}(X)$ coincides 
with that of $R_{\alpha}(X)^{*}=K[X] / \operatorname{in}\left(I_{\alpha}(X)\right)$ which is the Stanley-Reisner ring associated with the simplicial complex $\Delta_{\alpha}(X)$. We know that $\Delta_{\alpha}(X)$ is a shellable simplicial complex. Therefore, we may give a combinatorial interpretation of the $h$-vector of $R_{\alpha}(X)$ via the McMullen-Walkup formula. We need only to understand the set $C(Z)=\left\{x \in B\right.$ : there exists a facet $F$ of $\Delta_{\alpha}(X)$ such that $F<Z$ and $Z \backslash F=\{x\}$. We have seen that a facet $Z$ of the simplicial complex $\Delta_{\alpha}(X)$ is the union of disjoint sets $Z_{1}, \ldots, Z_{t}$ where $Z_{k}$ is a maximal chain of $X_{k}^{+}=$ $\left\{(i, j) \in B: \alpha_{k} \leq i \leq j\right\}$. We may interpret $Z_{k}$ as a path from a point of the set $\left\{\left(\alpha_{k}, \alpha_{k}\right),\left(\alpha_{k}+1, \alpha_{k}+1\right), \ldots,(n, n)\right\}$ to the point $\left(\alpha_{k}, n\right)$. Therefore the facets of $\Delta_{\alpha}(X)$ are families of non-intersecting paths. The following picture represents a facet of $\Delta_{\alpha}(X)$ where $\alpha=\{1,3\}$ and $n=5$.

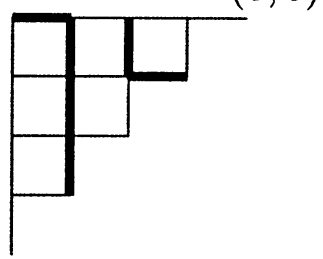

$(1,1)$

Fig. 3

By 1.11 , we have $C(Z)=C\left(Z^{\prime}\right)$, where by definition $Z^{\prime}=\min \{H: H$ is a facet of $\Delta_{\alpha}^{\prime}(X), H \cap B=Z$, and the minimum is taken with respect to the shelling of the facets of $\Delta_{\alpha}^{\prime}(X)$. Suppose that $Z$ is the family of non-intersecting paths $Z_{1}, \ldots$, $Z_{t}$ where $Z_{i}$ is a path from $\left(a_{i}, a_{i}\right)$ to $\left(a_{i}, n\right)$ with $\alpha_{i} \leq a_{i}$. Define $H_{i}$ to be the path from $\left(n, \alpha_{i}\right)$ to $\left(\alpha_{i}, n\right)$ obtaining from $Z_{i}$ by adding the set of points $\left\{\left(n, \alpha_{i}\right),\left(n-1, \alpha_{i}\right), \ldots,\left(a_{i}, \alpha_{i}\right),\left(a_{i}, \alpha_{i}+1\right), \ldots,\left(a_{i}, a_{i}\right)\right\}$. Then, from the definition of the shelling of $\Delta_{\alpha}^{\prime}(X)$, see [15, Th. 4.9], it is clear that $Z^{\prime}$ is the union of $H_{1}, \ldots, H_{t}$. In the following picture is represented the corresponding $Z^{\prime}$ of the facet in Fig. 3.

$(1,1)$

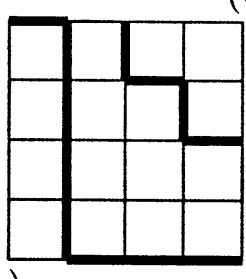

Fig. 4 
Given a path $P$ in $A$, a comer of $P$ is an element $(i, j) \in P$ for which $(i-1, j)$ and $(i, j-1)$ belong to $P$ as well. Let us denote by $c(P)$ the set of the corners of $P$. If $H$ is a facet of $\Delta_{\alpha}^{\prime}(X)$ and $H_{1}, \ldots, H_{t}$ is its decomposition as union of non-intersecting paths, then by, [5, 2.4], $C(H)=c\left(H_{1}\right) \cup \ldots \cup c\left(H_{t}\right)$. Thus, if $Z$ is a facet of $\Delta_{\alpha}(X)$, then $C(Z)$ is the set of the corners of $Z^{\prime}$. In our example of Fig. 3 and Fig. 4 we have $C(Z)=C\left(Z^{\prime}\right)=\{(2,5),(4,4)\}$.

Let $P$ be a path from $(b, b)$ to $(a, n)$ in the poset $B$, and let $(i, j)$ be a point of $P$. We define $(i, j)$ to be an $s$-comer of $P$ if $i<j$ and $(i-1, j),(i, j-1)$ belong to $P$, or $i=j$ (in this case $i=b$ ) and $(i-1, j)$ belongs to $P$. Let us denote by $\operatorname{sc}(P)$ the set of the s-corners of the path $P$, and if $Z$ is the family of non-intersecting paths $Z_{1}, \ldots, Z_{t}$ in $B$, define $\operatorname{sc}(Z)=\operatorname{sc}\left(Z_{1}\right) \cup \ldots \operatorname{sc}\left(Z_{t}\right)$. It is clear that the corners of $Z^{\prime}$ are exactly the s-corners of $Z$. Therefore we have:

Lemma 2.1. Let $Z$ be a facet of $\Delta_{\alpha}(X)$, then $C(Z)=\operatorname{sc}(Z)$.

Using the McMullen-Walkup formula, we obtain the following characterization of the $h$-vector of the ring $R_{\alpha}(X)$ :

Proposition 2.2. Let $\left(h_{0}, \ldots, h_{s}\right)$ be the $h$-vector of the ring $R_{\alpha}(X)$. Then $h_{i}$ is the number of families of non-intersecting paths $Z_{1}, \ldots, Z_{t}$ in $B$ with exactly $i$ s-comers, where $Z_{k}$ is a path from a point of the set $\left\{\left(\alpha_{k}, \alpha_{k}\right), \ldots,(n, n)\right\}$ to $\left(\alpha_{k}, n\right)$.

ExAmples 2.3. (a) Let $\alpha=1,3$ and $n=4$. In this case $I_{\alpha}(X)$ is the ideal generated by the 2 -minors of the first 2 rows and by all the 3 -minors of a $4 \times 4$ symmetric matrix of indeterminates. The non-intersecting paths are the following:
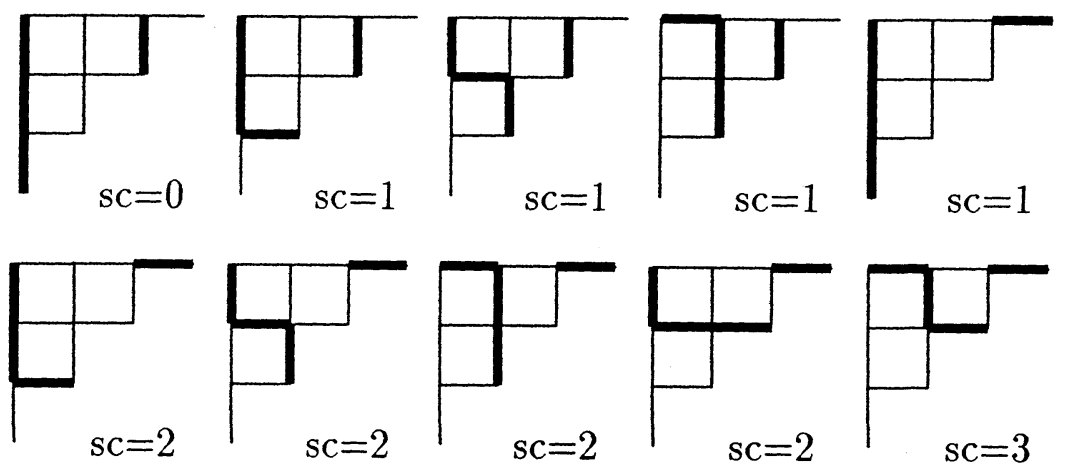

Fig. 5 
Hence the $h$-vector of $R_{\alpha}(X)$ is $(1,4,4,1)$.

(b) Consider the ring $R_{2}(X)$, and denote by $h_{0}(n), \ldots, h_{s}(n)$ its $h$-vector, where $n$ is the size of the matrix $X$. Then $h_{i}(n)$ is the number of paths from one point of the set $\{(1,1), \ldots,(n, n)\}$ to $(1, n)$ with $i$ s-corners.

The number of the paths with $i$ s-corners and which contain $(1, n-1)$ is $h_{i}(n-1)$. The number of those which contain $(3, n)$ is $h_{i}(n-1)-h_{i}(n-2)$. Finally, the number of those which contain $(2, n),(2, n-1)$ is $h_{i-1}(n-2)$. Thus we get $h_{i}(n)=2 h_{i}(n-1)-h_{i}(n-2)+h_{i-1}(n-2)$. By induction on $n, h_{i}(n)=\left(\begin{array}{c}n \\ 2 i\end{array}\right)$.

(c) Now consider the ring $R_{n-1}(X)$ and denote by $h_{0}(n), \ldots, h_{s}(n)$ its $h$-vector. By simple arguments as before one shows that $h_{i}(n)=2 h_{i-1}(n-1)-h_{i-1}(n-2)$ $+c(n)$, with $c(n)=1$ if $i \leq n-2$ and $c(n)=0$ otherwise. Then by induction, $h_{i}(n)=\left(\begin{array}{c}i+2 \\ 2\end{array}\right)$ if $i \leq n-2$ and $h_{i}(n)=0$ otherwise.

\subsection{The $a$-invariant of $R_{t}(X)$}

The $a$-invariant $a(S)$ of a positively graded Cohen-Macaulay $K$-algebra $S$ is the negative of the least degree of a generator of its graded canonical module. It can be read off from the Hilbert series $H_{S}(t)$ of $S$; more precisely $a(S)$ is the pole order of the rational function $H_{S}(t)$ at infinity.

For the computation of the $a$-invariant we restrict our attention to the ring $R_{t}(X)=K[X] / I_{t}(X)$, and we consider the weighted case too. Suppose there are given degrees to the indeterminates, say $\operatorname{deg} X_{i j}=v_{i j}$, such that the minors of $X$ are homogeneous. Then one has $2 v_{i j}=v_{i i}+v_{j j}$. Therefore essentially there are two possible degree types:

Type (a): There exist $e_{1}, \ldots, e_{n} \in \mathbf{N} \backslash\{0\}$ such that $\operatorname{deg} X_{i j}=e_{i}+e_{j}$ for all $1 \leq i \leq j \leq n$.

Type (b): There exist $e_{1}, \ldots, e_{n} \in \mathbf{N}$ such that $X_{i j}=e_{i}+e_{j}+1$ for all $1 \leq i \leq j$ $\leq n$.

Since the ideals under consideration are invariant under rows and columns permutations we may always assume $e_{1} \leq \cdots \leq e_{n}$.

Let us denote by $\Delta_{t}$ the simplicial complex $\Delta_{\alpha}(X)$, with $\alpha=\{1, \ldots, t-1\}$. The Hilbert function of $R_{t}(X)$ and $K\left[\Delta_{t}\right]=K[X] / \operatorname{in}\left(I_{t}(X)\right)$ coincide, thus we may as well compute the $a$-invariant of $K\left[\Delta_{t}\right]$. Since $\Delta_{t}$ is a shellable simplicial complex, Bruns-Herzog's proposition [5, 2.1] applies and we get: 
Theorem 2.4. Let $R=R_{t}(X)$. In the case of degree type (a):

$$
\begin{array}{ll}
a(R)=-(t-1)\left(\sum_{i=1}^{n} e_{i}\right) & \text { if } n \equiv t \bmod (2) \\
a(R)=-(t-1)\left(\sum_{i=1}^{n} e_{i}\right)-\sum_{i=1}^{t-1} e_{i} & \text { if } n \not \equiv t \bmod (2)
\end{array}
$$

And in the case of degree type (b):

$$
\begin{array}{ll}
a(R)=-(t-1)\left(\sum_{i=1}^{n} e_{i}+\frac{n}{2}\right) & \text { if } n \equiv t \bmod (2) \\
a(R)=-(t-1)\left(\sum_{i=1}^{n} e_{i}+\frac{n+1}{2}\right)-\sum_{i=1}^{t-1} e_{i} & \text { if } n \neq \equiv t \bmod (2)
\end{array}
$$

Proof. By $[5,2.1], a(R)=-\min \left\{\rho(Z): Z\right.$ is a facet of $\left.\Delta_{t}\right\}$, where

$$
\rho(Z)=\sum_{(i, j) \in Z \backslash C(Z)} \operatorname{deg} X_{i j} .
$$

We define a facet $F$ of $\Delta_{t}$ and prove that $\rho(F) \leq \rho(Z)$ for all the facets $Z$ of $\Delta_{t}$. Then the desired result will follow from the computation of $\rho(F)$.

For $i=1, \ldots, t-2$, let $D_{i}$ be the set $\{(i, n),(i, n-1), \ldots,(i, n-t+i+$ 2)\}, and set $D_{t-1}=\emptyset$.

If $n \equiv t \bmod (2)$, we define $F_{i}$ to be the path from $(i, n)$ to $((n-t) / 2+i+$ $1,(n-t) / 2+i+1)$ which is obtained from $D_{\imath}$ by adding the points $(i, n-t$ $+i+1),(i+1, n-t+i+1), \ldots, \quad(i+j, n-t+i-j+1), \quad(i+j+1$, $n-t+i-j+1), \ldots,(i+(n-t) / 2,(n-t) / 2+i+1),((n-t) / 2+i+1$, $(n-t) / 2+i+1)$.

If $n \not \equiv t \bmod (2)$, we define $F_{i}$ to be the path from $(i, n)$ to $((n-t+1) / 2$ $+i,(n-t+1) / 2+i)$ which is obtained from $D_{i}$ by adding the points $(i$, $n-t+i+1),(i, n-t+1),(i+1, n-t+i), \ldots,(i+j, n-t+i-j)$, $(i+j+1, n-t+i-j), \ldots,(i+(n-t-1) / 2,(n-t+1) / 2+i),((n-$ $t+1) / 2+i,(n-t+1) / 2+i)$.

Finally we define $F$ to be the family of non-intersecting paths $F_{1}, \ldots, F_{t-1}$. The following picture illustrates $F$ when $t=4$ and $n=8,9$. 

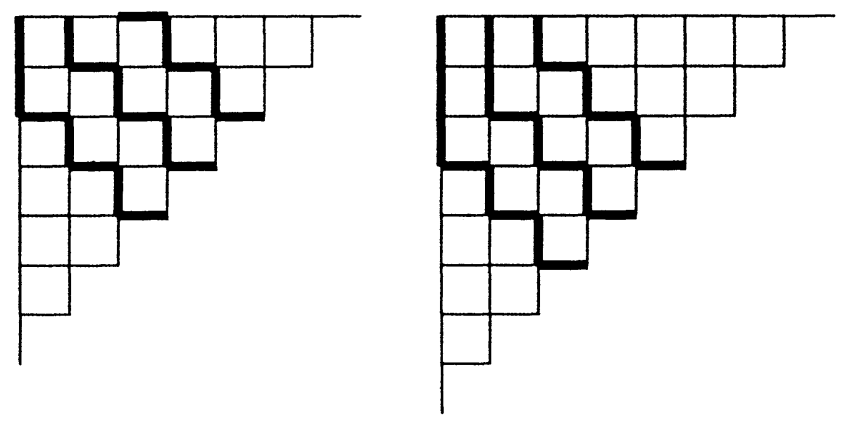

Fig. 6

We start considering $t=2$ and $n$ even. In this case $C(F)=\{(2, n),(3, n-$ $1), \ldots,(n / 2+1, n / 2+1)\}$, and therefore $F \backslash C(F)=\{(1, n),(2, n-1)$, $\ldots,(n / 2, n / 2+1)\}$. One has $\rho(F)=\sum_{i=1}^{n} e_{i}$ or $\rho(F)=\sum_{i=1}^{n} e_{i}+n / 2$ if the degree is of type (a) or (b), respectively. Given $Z$ a path from $(1, n)$ to $(p, p)$ we claim that for all $i<p$ there exists $j$ such that $(i, j) \in Z \backslash C(Z)$, and that for all the $i \geq p$ there exists $j$ such that $(j, i) \in Z \backslash C(Z)$. From the claim it follows easily that $\rho(Z) \geq \rho(F)$. To prove the claim observe that if $i<p$ (resp. $i \geq p$ ) then there exists $j$ such that $(i, j) \in Z$ (resp. $(j, i) \in Z$ ), and if $(i, j) \in$ $C(Z)$, then $(i, j-1) \in Z \backslash C(Z)$ (resp. if $(j, i) \in C(Z)$, then $(j-1, i) \in Z \backslash$ $C(Z))$.

If $t=2$ and $n$ is odd, we have $\rho(F)=\sum_{i=1}^{n} e_{i}+e_{1}$ or $\rho(F)=\sum_{i=1}^{n} e_{i}+e_{1}$ $+(n+1) / 2$. Let $Z$ be a path from $(1, n)$ to $(p, p)$. Since $n$ is odd we deduce from the previous claim that $|Z \backslash C(Z)| \geq(n+1) / 2$, and that there exists $i$ which appears twice as a coordinate of some elements in $Z \backslash C(Z)$. By assumption $e_{1} \leq e_{2} \leq \ldots \leq e_{n}$, therefore $\rho(F) \leq \rho(Z)$.

Now let $t \geq 2$ and let $Z$ be a facet of $\Delta_{t}$, that is a family of non-intersecting paths $Z_{1}, \ldots, Z_{t-1}$. Since the paths are non-intersecting, $D_{k} \subset Z_{k}$ for all $k=$ $1, \ldots, t-1$. We may think of $F_{k}$ and $Z_{k}$ as paths starting from $(i, n-t+i+1)$, and argue as before to show that:

$$
\sum_{(i, j) \in F_{k} \backslash S C\left(F_{k}\right)} \operatorname{deg} X_{i j} \leq \sum_{(i, j) \in Z_{k} \backslash S C\left(Z_{k}\right)} \operatorname{deg} X_{i j}
$$

for all $k=1, \ldots, t-1$. Therefore we get:

$$
\rho(F)=\sum_{k=1}^{t-1} \sum_{(i, j) \in F_{k} \backslash S C\left(F_{k}\right)} \operatorname{deg} X_{i j} \leq \sum_{k=1}^{t-1} \sum_{(i, j) \in Z_{k} \backslash S C\left(Z_{k}\right)} \operatorname{deg} X_{i j}=\rho(Z)
$$


and we are done.

The homogeneous case (all the indeterminates have degree 1) arises from a degree type (b) with $e_{i}=0$ for all $i$. Therefore

$$
a\left(R_{t}(X)\right)= \begin{cases}-(t-1) \frac{n}{2} & \text { if } n \equiv t \bmod (2) \\ -(t-1) \frac{n+1}{2} & \text { if } n \neq \equiv t \bmod (2) .\end{cases}
$$

By a result of Goto [3], $R_{t}(X)$ is Gorenstein if and only if $n \equiv t \bmod (2)$. If $n \not \equiv t \bmod (2)$, the canonical module of $R_{t}(X)$ is the prime ideal $P$ generated by all the $t-1$ minors of the first $t-1$ rows of $X$. It is not difficult to see that, up to shift, $P$ is also the graded canonical module of $R_{t}(X)$. Hence the graded canonical $\omega_{t}$ module of $R_{t}(X)$ is:

$$
\omega_{t}= \begin{cases}R_{t}(X)\left(-(t-1) \frac{n}{2}\right) & n \equiv t \bmod (2) \\ P\left(-(t-1) \frac{n-1}{2}\right) & n \neq t \bmod (2)\end{cases}
$$

\subsection{Determinantal rings associated with a matrix in which a submatrix is symmetric}

Let $Z=\left(Z_{i j}\right)$ be an $m \times n$ matrix, $m \leq n$, whose entries are indeterminates such that the submatrix of the last $s$ rows and of the first $s$ columns is symmetric, with $s>1$. Using the blocks notation, we write:

$$
Z=\left(\begin{array}{cc}
M & N \\
S & P
\end{array}\right)
$$

where $M=\left(M_{i j}\right), N=\left(N_{i j}\right), P=\left(P_{i j}\right)$ are generic matrices of indeterminates of size $(m-s) \times s,(m-s) \times(n-s), s \times(n-s)$, respectively, and $S=$ $\left(S_{i j}\right)$ is an $s \times s$ symmetric matrix of indeterminates. Denote by $K[Z]$ the polynomial ring over the field $K$ whose indeterminates are the entries of $Z$.

Let $I_{t}(Z)$ be the ideal generated by all the t-minors of $Z$ and denote by $R_{t}(Z)$ the ring $K[Z] / I_{t}(Z)$. If $s=m$, then $Z$ is called a partially symetric matrix. When $Z$ is partially symmetric, $R_{t}(Z)$ is essentially a ring of the class $R_{\alpha}(X)$, see $[8,2.5]$.

Next we will interpret $R_{t}(Z)$ as a ladder determinantal ring. To do this, we 
take two symmetric matrices of distinct indeterminates $E_{1}, E_{2}$, of size $(m-s) \times$ $(m-s),(n-s) \times(n-s)$. We construct an $(m+n-s) \times(m+n-s)$ symmetric matrix of indeterminates in the following way:

$$
X=\left(\begin{array}{ccc}
E_{1} & M & N \\
M^{t} & S & P \\
N^{t} & P^{t} & E_{2}
\end{array}\right)
$$

Denote $A=\left\{(i, j) \in \mathbf{N}^{2}: 1 \leq i, j \leq m+n-s\right\}$, and $V=\{(i, j) \in A: i \leq m$ and $j>m-s\}$. $V$ is the semi-symmetric ladder of $X$ corresponding to $Z$. The set $L=\{(i, j) \in A: i>m-s$ or $j<n-s\}$ is the symmetric ladder associated with $V$. Let $\alpha=\{1, \ldots, t-1\}$, then by construction and by 1.5 we have $I_{\alpha}(L)=I_{\alpha}(V)=I_{t}(Z)$ and $R_{t}(Z)=R_{\alpha}(L)$. Let us denote by $\Delta_{t}(Z)$ the simplicial complex $\Delta_{\alpha}(L)$.

Let $\tau^{\prime}$ be the lexicographic term order on the monomials of $K[Z]$ induced by the variable order which is obtained listing the entries of $Z$ as they appear row by row. Let $J$ be the set of all the minors $\left[a_{1}, \ldots, a_{t} \mid b_{1}, \ldots, b_{t}\right]$ of $Z$ (the indices refer to $Z$ and not to $X$ ) such that $b_{i}-a_{\imath} \geq-m+s$. In other words $J$ is the set of the $t$-minors of $Z$ whose main diagonal does not lie under the main diagonal of $S$. By 1.3 and 1.13 , it follows immediately:

Proposition 2.5. (a) The ring $R_{t}(Z)$ is a Cohen-Macaulay domain. (b) $J$ is a minimal system of generators of $I_{t}(Z)$ and a Gröbner basis with respect to $\tau^{\prime}$.

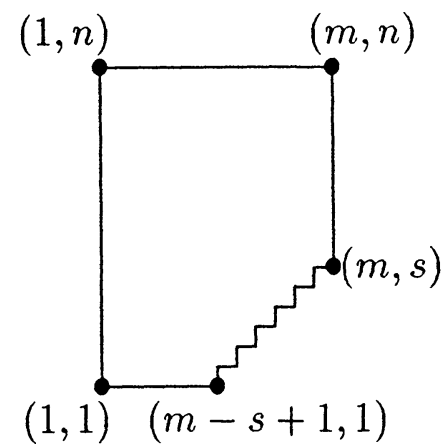

Fig. 7

In order to compute the dimension and multiplicity of $R_{t}(Z)$, we describe the simplicial complex $\Delta_{t}(Z)$. It seems more natural to use the labelling of $Z$ instead of that of $X$, so that we can identify $L^{+}$with the set $\left\{(i, j) \in \mathbf{N}^{2}: 1 \leq i \leq m, 1 \leq j\right.$ 
$\leq n, j-i \geq s-m\}$, see FIg. 7. Note that, in this case, $L_{\imath}^{+}$is obtained from $L_{i-1}^{+}$ by deleting the lower border. Thus, if $i \leq s$, then $\operatorname{rk}\left(L_{i}^{+}\right)=(n+m-s+1-i)$, and if $i \geq s$, then $\operatorname{rk}\left(L_{i}^{+}\right)=(n+m-2 i+1)$. Therefore, from 1.9, we get:

If $t \geq s$, then

$$
\operatorname{dim} R_{t}(Z)=(n+m+1-t)(t-1)-\frac{s(s-1)}{2} .
$$

The dimension of the determinantal ring $R_{t}\left(X_{1}\right)$ associated with the ideal of the $t$-minors of an $m \times n$ generic matrix of indeterminates $X_{1}$ is $(n+m+1-t)$ $(t-1)$, see [7, Cor. 5.12]. Therefore $R_{t}(Z)$ is nothing but a specialization of $R_{t}\left(X_{1}\right)$, that is $R_{t}(Z)$ is isomorphic to $R_{t}\left(X_{1}\right) / I$ where $I$ is the ideal generated by the regular sequence of the $s(s-1) / 2$ linear forms which give the symmetry relations on $Z$. Moreover, $R_{t}(Z)$ and $R_{t}\left(X_{1}\right)$ have the same multiplicity and the same $h$-vector.

If $t<s$, then:

$$
\operatorname{dim} R_{t}(Z)=\left(n+m+1-s-\frac{1}{2}\right)(t-1) .
$$

In this case we can interpret a facet of $\Delta_{t}(Z)$ as a family of non-intersecting paths $H_{1}, \ldots, H_{t-1}$ where $H_{i}$ is a path from one point of the set $\{(m-s+1,1)(m-s$ $+2,2), \ldots,(m, s)\}$ to $(i, n)$. Let us denote by $P_{i}=(i, n)$ and $Q_{j}=(m-s+$ $j, j$ ). Given $1 \leq j_{1}<\ldots, j_{t-1} \leq s$, according to [19, Sect. 2.7], the number of families of non-intersecting paths from $Q_{j_{1}}, \ldots, Q_{j_{t-1}}$ to $P_{1}, \ldots, P_{t-1}$ is $\operatorname{det}\left(W\left(P_{h}\right.\right.$, $\left.\left.Q_{j_{k}}\right)\right)_{1 \leq h, k \leq t-1}$ where $W\left(P_{h}, Q_{j_{k}}\right)$ is the number of paths from $P_{h}$ to $Q_{j_{k}}$. But it is easy to see that

$$
W\left(P_{h}, Q_{j_{k}}\right)=\left(\begin{array}{c}
n+m-s-h \\
n-j_{k}
\end{array}\right) .
$$

Hence we get the following formula for the multiplicity of $R_{t}(Z)$ :

$$
e\left(R_{t}(Z)\right)=\sum_{1 \leq j_{1}<\ldots<j_{t-1} \leq s} \operatorname{det}\left[\left(\begin{array}{c}
n+m-s-h \\
n-j_{k}
\end{array}\right)\right]_{1 \leq h, k \leq t-1} .
$$

As we did for the ring $R_{\alpha}(X)$, we may give a combinatorial interpretation of the $h$-vector $R_{t}(Z)$ in terms of number of non-intersecting paths with a fixed number of certain corners. The case $t \geq s$, by the above discussion, is solved in [5].

Suppose $t<s$. A facet $H$ of $\Delta_{t}(Z)$ is a family of non-intersecting paths $H_{1}, \ldots, H_{t-1}$, where $H_{i}$ is a path from one point of set $\{(m-s+i, i),(m-s+$ 
$i+1, i+1), \ldots,(m, s)\}$ to $(i, n)$. We distinguish two cases:

If $s=m$, then $C(H)=\operatorname{sc}\left(H_{1}\right) \cup \ldots \cup \mathrm{sc}\left(H_{t-1}\right)$. This follows from the fact that when we consider $\Delta_{t}(Z)$ as a sub-complex of $\Delta_{t}(X)$, it has the following property: if $H$ is a facet of $\Delta_{t}(Z)$ and $H_{1} \in \Delta_{t}(X)$ with $H_{1}<H$ in the shelling of $\Delta_{t}(X)$ and $H \backslash H_{1}=\{(a, b)\}$, then $H_{1} \in \Delta_{t}(Z)$. Therefore, if we denote by $h_{i}$ the number of families of non-intersecting paths with exactly $i$ s-corners, $\left(h_{0}, \ldots\right.$, $\left.h_{s}\right)$ is the $h$-vector of $R_{t}(Z)$.

If $s<m$, then $C(H)=\left(\operatorname{sc}\left(H_{1}\right) \backslash\left\{T_{1}\right\}\right) \cup \ldots \cup\left(\operatorname{sc}\left(H_{t-1}\right) \backslash\left\{T_{t-1}\right\}\right)$, where $T_{i}$ is the point $(m-s+i, i)$. This follows from the fact that when we consider $\Delta_{t}(Z)$ as a subcomplex of $\Delta_{t}(Z)$, if $H_{1}$ is a facet of $\Delta_{t}(X)$ such that $H_{1}<H$ and $H \backslash H_{1}=\{(a, b)\}$ then $H_{1}$ is in $\Delta_{1}(Z)$ unless $(a, b)=T_{i}$ for some $i$ and $T_{i}$ belongs to $H_{i}$.

For instance, consider the case in which $t=4, s=5, m=n=6$. The two facets $H$ and $K$ in the following picture have s-corners respectively in $\{(2,1)$, $(3,2),(5,6),(6,5)\}$, and $\{(2,3),(3,2),(5,4),(5,6),(6,5)\}$. It is clear from the picture that it is not possible to find a family of paths which differs from $H$ only in (3.2) and that is earlier in the shelling. The point $T_{1}=(2,1)\left(\mathrm{resp} . T_{2}=\right.$ $(3,2)$ ) is not in $C(H)$ since it is an s-corner of $H_{1}$ (resp. $H_{2}$ ). The point $(3,2)$ is in $C(K)$ since it is an s-corner but not of $K_{2}$. Hence $C(H)=\{(5,6),(6,5)\}$, and $C(K)=\{(2,3),(3,2),(5,4),(5,6),(6,5)\}$.
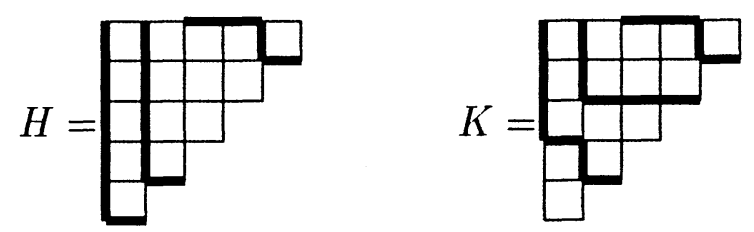

Fig. 8

Therefore, if we denote by $h_{i}$ the number of families of non-intersecting paths $H$ with $\left|\left(\operatorname{sc}\left(H_{1}\right) \backslash\left\{T_{1}\right\}\right) \cup \ldots\left(\operatorname{sc}\left(H_{t-1}\right) \backslash\left\{T_{t-1}\right\}\right)\right|=i$, then $\left(h_{0}, \ldots, h_{s}\right)$ is the $h$-vector of $R_{t}(Z)$.

EXAmples 2.6. From the computation of the $h$-vector of $R_{2}(X)$ it follows immetiately: (a) If $s=m$ and $n=m+1$, then $h_{i}\left(R_{2}(Z)\right)=\left(\begin{array}{c}n \\ 2 i\end{array}\right)$ if $i \neq 1$, and $h_{1}\left(R_{2}(Z)\right)=\left(\begin{array}{l}n \\ 2\end{array}\right)-1$. 
(b) If $s+1=m=n$, then $h_{i}\left(R_{2}(Z)\right)=\left(\begin{array}{c}n+1 \\ 2 i\end{array}\right)$ if $i \neq 1$, and $h_{1}\left(R_{2}(Z)\right)=$ $\left(\begin{array}{c}n+1 \\ 2\end{array}\right)-2$

If $s=m<n$, then the ring $R_{t}(Z)$ is essentially one of the class $R_{\alpha}(X)$, and in [10] we proved that it is always normal and that is Gorenstein if and only if $2 m=n+t$. We now show:

THEOREM 2.7. Let $s<m$, then (a) $R_{t}(Z)$ is a normal domain. (b) $R_{t}(Z)$ is Gorenstein if and only if $t \geq s$ and $m=n$.

Proof. (a) Let us consider the following two symmetric ladders of $X: L_{1}=$ $\{(i, j) \in A: i>m-s$ or $j>m-s\}, L_{2}=\{(i, j) A: i<n-s$ or $j<n-s\}$. The ladder determinantal rings $R_{t}\left(L_{1}\right), R_{t}\left(L_{2}\right)$ are the determinantal rings associated with the partially symmetric matrices $Z_{1}$ and $Z_{2}$, where:

$$
Z_{1}=\left(\begin{array}{ll}
M & N \\
S & P \\
P^{t} & E_{2}
\end{array}\right) \quad Z_{2}=\left(\begin{array}{lll}
E_{1} & M & N \\
M^{t} & S & P
\end{array}\right)
$$

Denote by $Y_{i}$ the support of $L_{i}$. The set of the doset $t$-minors is a Gröbner basis of $I_{t}(X)$. Then the set $B(X)$ of the monomials in the set of indeterminates $X_{i j}$, $1 \leq i \leq j \leq n+m-s$, which are not divisible by leading terms of $t$-minors form a $K$-basis of the ring $R_{t}(X)$. For the same reason the subset $B\left(Y_{i}\right)$ of $B(X)$ of the monomials in the set $Y_{i}$ not divisible by leading terms of doset $t$-minors form a $K$-basis of the ring $R_{t}\left(L_{i}\right)$. A $K$-basis of $R_{t}\left(L_{1}\right) \cap R_{t}\left(L_{2}\right)$ is $B\left(Y_{1}\right) \cap B\left(L_{2}\right)$, but the last is also a $K$-basis of $R_{t}(Z)$. Hence $R_{t}(Z)=R_{t}\left(L_{1}\right) \cap$ $R_{t}\left(L_{2}\right)$, and we conclude that $R_{t}(Z)$ is normal since $R_{t}\left(L_{1}\right)$ and $R_{t}\left(L_{2}\right)$ are.

(b) If $t \geq s$ and $m=n$, then $R_{t}(Z)$ is Gorenstein since it is a specialization of a Gorenstein ring, [7, 8.9].

To prove the converse we argue by induction on $t$. Let $t=2$; consider the residue class $x$ of $N_{1 n-s}$ in $R_{2}(Z)$, and denote by $D$ the set of the residue classes of the indeterminates in the first row and last column of $Z$, that is $M_{11}, \ldots, M_{1 s}$, $N_{11}, \ldots, N_{1 n-s}, \ldots, N_{m-s n-s}, P_{1 n-s}, \ldots, P_{s n-s}$. Let $K[D]$ be the $K$-subalgebra of $R_{2}(Z)$ generated by $D$.

It is clear that $K[D]\left[x^{-1}\right]=R_{2}(Z)\left[x^{-1}\right]$. Furthermore, we have the following relations $M_{1 i} P_{j n-s}=S_{j i} N_{1 n-s}=S_{i j} N_{1 n-s}=M_{1 j} P_{i n-s} \bmod I_{2}(Z)$, for all $1 \leq$ $i, j \leq s$. By dimension considerations, $K[D]\left[x^{-1}\right]$ is isomorphic to the polynomial ring 


$$
R\left[N_{11}, \ldots, N_{1 n-s}, \ldots, N_{m-s n-s}\right]\left[N_{1 n-s}^{-1}\right]
$$

over the ring $R$, where

$$
R=K\left[M_{11}, \ldots, M_{1 s}, P_{1 n-s}, \ldots, P_{s n-s}\right] / I,
$$

and $I$ is the ideal generated by the 2 minors of the matrix

$$
\left(\begin{array}{lll}
M_{11} & \ldots & M_{1 s} \\
P_{1 n-s} & \ldots & P_{s n-s}
\end{array}\right) .
$$

By assumption $R_{2}(Z)$ is Gorenstein. Therefore $R_{2}(Z)\left[x^{-1}\right]$ is Gorenstein and $R$ is Gorenstein too. But this is possible only if $s=2,[7,8.9]$. Then $R_{2}(Z)$ is a specialization of the determinantal ring associated with the ideal of the 2 -minors of a generic $m \times n$ matrix. Therefore, by [7, 8.9], $m=n$. If $t>2$, we apply the usual inversion trick. After inversion of $s_{11}$ the residue class of $S_{11}$, we get an isomorphism between $R_{t}(Z)\left[s_{11}^{-1}\right]$ and $R_{t-1}\left(Z_{1}\right)\left[T_{1}, \ldots, T_{m+n-s}\right]\left[T_{1}^{-1}\right]$, where the $T_{i}$ are indeterminates and $Z_{1}$ is an $m-1 \times n-1$ matrix of indeterminates such that the submatrix of the last $s-1$ rows and first $s-1$ columns is symmetric (when $s=2, Z_{1}$ is generic). Since $R_{t}(Z)$ is Gorenstein, $R_{t-1}\left(Z_{1}\right)$ is Gorenstein and, by induction, $s-1 \leq t-1$ and $m-1=n-1$. Therefore $s \leq t$ and $n=m$.

\section{REFERENCES}

[1] Abhyankar S. S., Enumerative combinatorics of Young tableaux, Marcel Dekker, New York, 1988.

[2] Abhyankar S. S., Kulkarni D. M., On Hilbertian ideals, Linear Algabra and its Appl., 116 (1989), 53-79.

[3] Barile M., The Cohen-Macaulayness and the $a$-invariant of an algebra with straightening law on a doset, Comm. in Alg., 22 (1994), 413-430.

[4] Björner A., Shellable and Cohen-Macaulay partially ordered sets, Trans. Amer. Math. Soc., 260 (1980), 159-183.

[5] Bruns W., Herzog J., On the computation of $a$-invariants, Manuscripta Math., 77 (1992), 201-213.

[6] W. Bruns, J. Herzog, U. Vetter, Syzygies and walks, to appear in the Proc. of the Workshop in Comm. Alg. Trieste 1992.

[7] Bruns W., Vetter U., Determinantal rings, Lect. Notes Math. 1327, Springer, Heidelberg, 1988.

[8] Conca A., Gröbner bases of ideals of minors of a symmetric matrix, J. of Alg., 166 (1994), 406-421.

[9] - Ladder determinantal rings, to appear in J. of Pure and Appl. Alg.

[10] - Divisor class group and canonical class of determinantal rings defined by ideals of minors of a symmetric matrix, Arch. Mat. 63 (1994), 216-224. 
[11] Conca A., Herzog J., On the Hilbert function of determinantal rings and their canonical module, to appear in Proc. Amer Math. Soc.

[12] De Concini C., Eisenbud D., Procesi C., Hodge algebras, Asterisque 91, 1982.

[13] Goto S., On the Gorensteinness of determinantal loci, J. Math. Kyoto Univ., 19 (1979), 371-374.

[14] Gräbe H. G., Streckungsringe, Dissertation B, Pädagogische Hochschule Erfurt, 1988.

[15] Herzog J., Trung N. V., Gröbner bases and multiplicity of determinantal and pfaffian ideals, Adv. Math., 96 (1992), 1-37.

[16] Kutz R., Cohen-Macaulay rings and ideal theory in rings of invariants of algebraic groups, Trans. Amer. Math. Soc., 194 (1974), 115-129.

[17] Narasimhan H., The irreducibility of ladder determinantal varieties, J. Alg., 102 (1986), 162-185.

[18] Stanley R., Combinatorics and Commutative Algebra, Birkhäuser, Basel, 1983.

[19] - Enumerative Combinatorics I, Wardworth and Brook, California, 1986.

FB6 Mathematik und Informatik

Universität GHS Essen

45117 Essen, Germany 